\title{
Conceptual Model for Behaviour Change Progress - Instrument in Design Processes for Behaviour Change Systems
}

\author{
Helena LINDGREN ${ }^{1}$ and Saskia WECK \\ Department of Computing Science, Umeå University, Umeå, Sweden
}

\begin{abstract}
The aim of the work presented in this article was to develop a conceptual model for behavior change progress, which could be used for automated assessment of reasons for progress or non-progress. The model was developed based on theories for behavior change, and evaluated by domain experts. The information models of two prototype systems of a digital coach under development for preventing cardiovascular diseases and stress respectively, were evaluated by comparing the content of the prototypes with concepts in the model. The conceptual model was found useful as instrument to evaluate to what extent the prototypes are based in theories for behavior change, whether some vital information is missing, and to identify mechanisms for short and long time goal setting. Moreover, the connection between the ontology underpinning the prototypes and the conceptual model could be defined. Future work includes the integration of the conceptual model to function as a metaontology, which could be used for capturing causal relationships between information collected by the applications at baseline and at runtime.
\end{abstract}

Keywords. behavior change, ontology, participatory design, knowledge engineering, personalisation

\section{Introduction}

Theories of behavior change (e.g. [1-5]), and design models for behavior change systems, partly based on these theories [6-7], are increasingly applied in research on persuasive technology for behavior change [8]. When applying a participatory design process to develop such systems, a central assumption is that the participating stakeholders are experts in the domain in which the persuasive technology is aimed to be used. The design process aims to translate this knowledge into digital tools, which an individual can use for health coaching. Moreover, the knowledge needs to be translated into formal, computational models, which the system can use for tailoring support and automatically and intelligently adapt its behavior to the individual (e.g. [9-10]).

The purpose of this study is to develop and evaluate a conceptual model of behavior change progress, which can be used in a participatory design process of behavior change systems, and for automated analysis of progress over time as a complement to everyday assessments at runtime.

${ }^{1}$ Corresponding Author, Helena Lindgren, Department of Computing Science, Umeå University, 90187 Umeå, Sweden; E-mail: helena.lindgren@umu.se. 


\section{Methods}

Theories on behavior change explain the determinants of human behavior, motivations for behavior, and how to guide behavior change. The conceptual model is based on the Theory of Planned Behavior [1], the Self-Determination Theory [2], Prochaska's Transtheoretical Model of Change [3], the Goal Setting Theory [4], and the Fogg Behavior Model [6]. Concepts from the Health Belief Model [5] were also embedded.

The model was created in the following steps [11]. Relevant concepts were selected from behavior change theories, and defined. Next, based on the theoretical frameworks, the relationships between the concepts were defined and explained. In a third step, the initial model was validated by five experts through interviews and adjusted accordingly. Participating experts represented expertise in epidemiology, nutrition, sociology and nursing. The model was applied and further evaluated as part of two ongoing participatory design processes [10,12]. One aims at developing a digital coach application for preventing cardio-vascular diseases and promoting change of behavior to improve health [13], another to support stress rehabilitation. The preliminary information models of the two prototypes, which had emerged in focus group discussions and workshops, were evaluated by connecting the information to the conceptual model.

\section{Results}

The experts interviewees suggested changes of the conceptual model based on their knowledge of the concepts, theories and experience from clinical practice, gave elaborations on the concepts, and proposed additional concepts and relationships, partly based on the Health Belief Model (e.g., Risk Perception, Previous Attempts) [5,11].

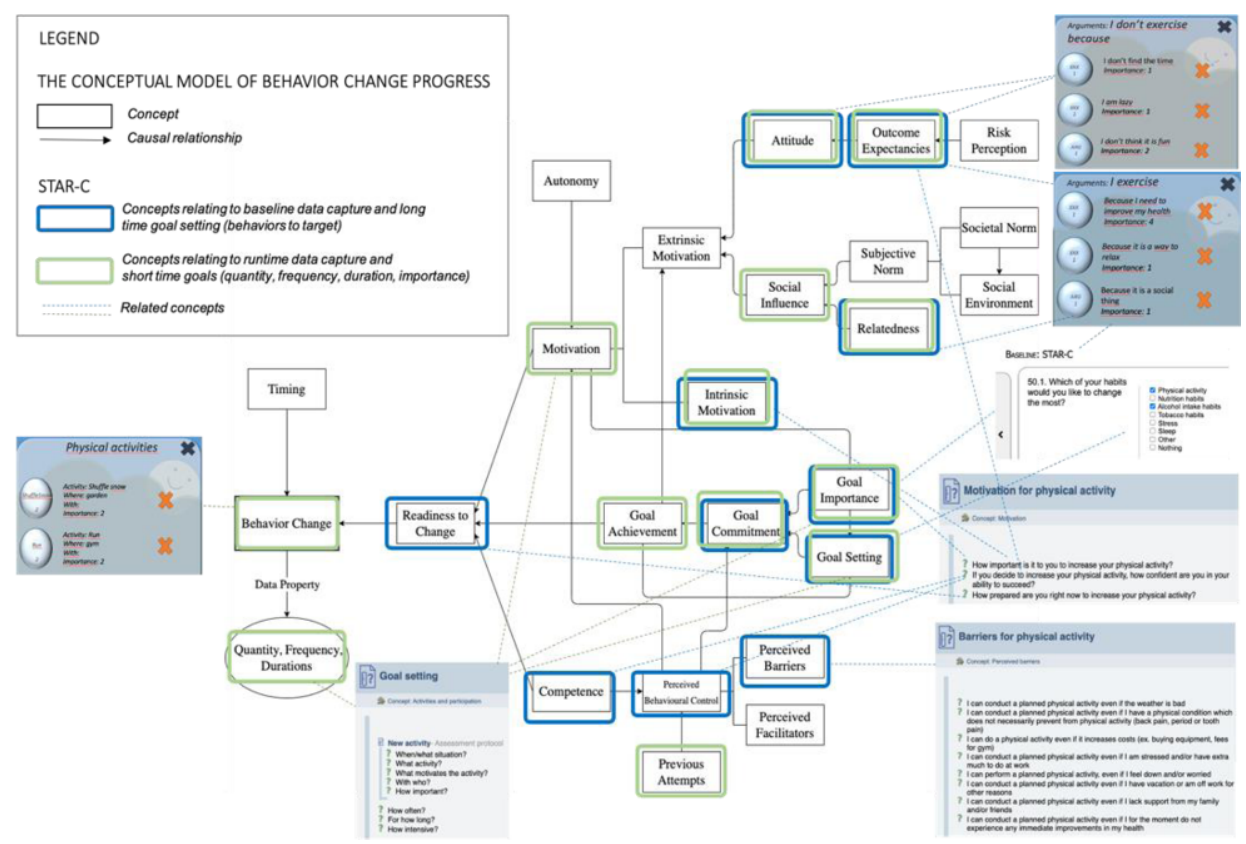

Figure 1. STAR-C information mapped to the conceptual model of behavior change progress. 
The baseline questions for the cardio-vascular intervention cover most of the factors relating to a person's own attitude and intention to change, while less information is collected on how the social environment may influence the factors for behavior change. The runtime version contains mechanisms that may capture also conflicting motives for activities and more on perceived barriers, which are expected to illuminate reasons for non-adherence to goals specified, and be used for explaining and motivating changes in behaviors.

When mapping the contents of the baseline and ecological momentary assessment (EMA) questions for the coaching system for managing stress (Jonglera) to the conceptual model, it is clear that stress management requires a different approach to design than the previous example, in particular, since mobilizing the physical and mental effort required to changing behavior is part of the behavior change progress, and targeted as part of the digital intervention (Figure 2). In this case, the EMA questions are posed to assess daily engagement and experiences, forming a basis for the system to translate this into motivating trajectories of positive trends, which can be communicated to the person as an extrinsic motivator. As such, it will target also the factors in the behavior change progress model such as outcome expectancies, attitudes, barriers and facilitators.

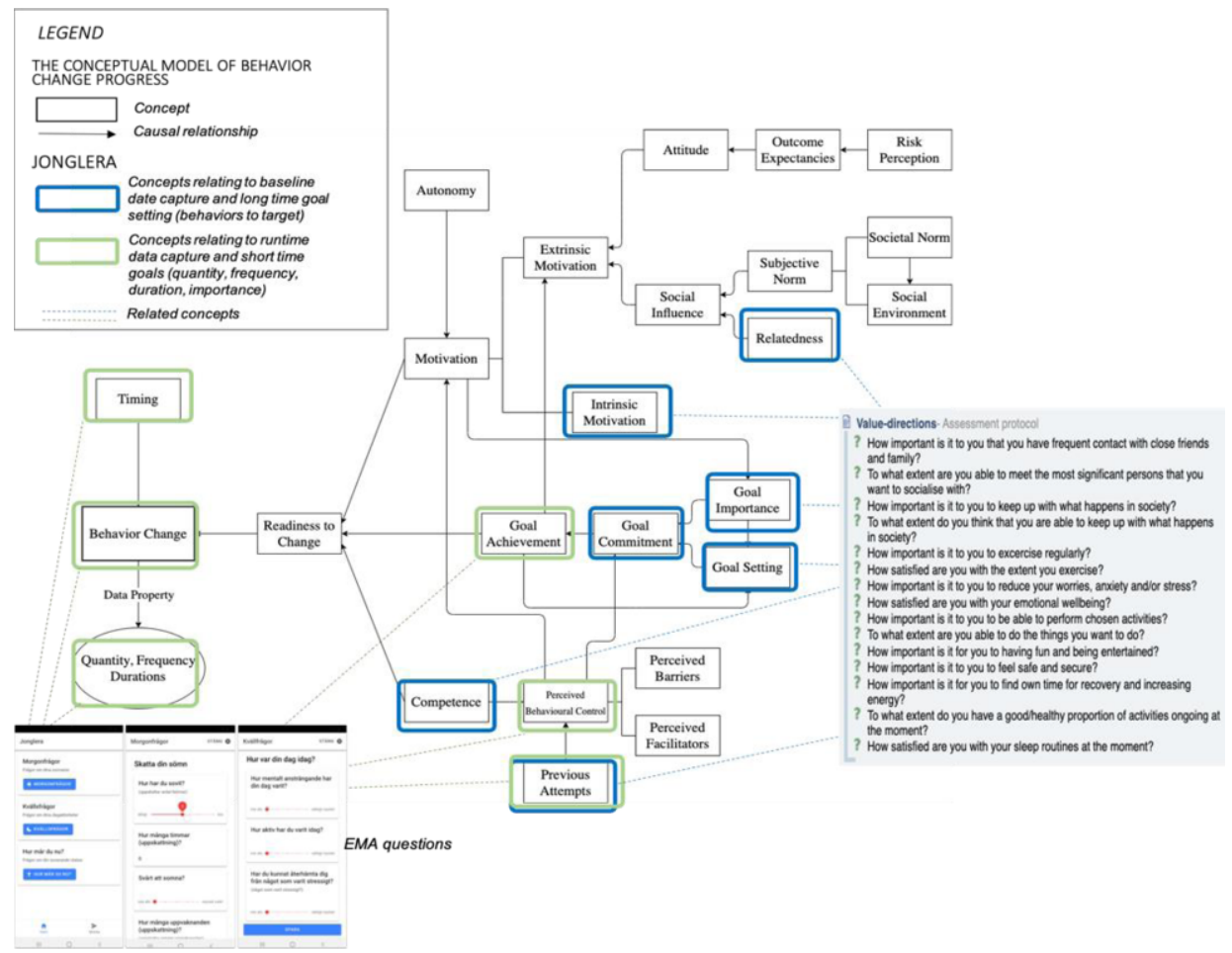

Figure 2. Jonglera information mapped to the conceptual model of behavior change progress.

To summarize, the conceptual model illuminates the complexity of behavior change for improving emotional wellbeing relating to stress and exhaustion syndrome, and the importance to design mechanisms, which can translate small efforts into positive outcome expectancies and attitudes, which can in turn, reinforce sense of competence and self-efficacy. 


\section{Discussion and Conclusions}

A conceptual model of behavior change progress was developed based on theories of behavior change, evaluated by experts on behavior change and applied to evaluate the information models of two prototype systems for supporting behavior change. It was concluded that the model is useful for assessing to what extent an information model is capturing vital factors for behavior change, and how personalized intervention needs to be designed in order to promote behavior change.

Ontologies have been developed for different purposes, e.g., behavior change interventions, or identifying barriers for change [14-15]. The proposed conceptual model for behavior change progress aims to capture the vital concepts of the relevant theories, and their relationships in the development of persuasive systems. As such, the model complements existing models.

Further evaluation studies need to be done, in particular for exploring how the model can be used as instrument by the participating domain experts and stakeholders in a design process. Future work includes also the integration of the conceptual model to function as a meta-ontology in the system architectures of the two prototype systems, which could be used for capturing causal relationships between information collected by an application at baseline and at runtime.

\section{References}

[1] Ajzen I. The theory of planned behavior. Organizational Behavior and Human Decision Processes 1991;50:179-211.

[2] Ryan RM, Deci LM. Self-Determination Theory and the facilitation of intrinsic motivation, social development and well-being. American Psychologist 2000;55:68-78.

[3] Prochaska JO, Redding CA, Evers KE, The transtheoretical model and stages of change. In: Glanz A, Rimer BK, Viswanath K (Edrs.) Health behavior and health education: Theory, research, and practice. pp. 97-121. Jossey-Bass; 2008.

[4] Locke EA, G.P. Latham GP. Goal setting theory. In: Motivation: Theory and Research 2012:23-40.

[5] Janz NK, Becker MH. The Health Belief Model: A Decade Later. Health Education Quarterly 1984;11,1: $1-47$.

[6] Fogg BJ. A behaviour model of persuasive design, In Proc. of the $4^{\text {th }}$ International Conference on Persuasive Technology (Persuasive'09). Association for Computing Machinery, New York, NY, USA, 2009:1-7.

[7] Oinas-Kukkonen H, Harjumaa M. Persuasive Systems Design: Key Issues, Process Model, and System Features. Communnications for the Association for Information Systems 2009;24,28: 485-500.

[8] Orji R, Moffatt K. Persuasive technology for health and wellness: State-of-the-art and emerging trends. Health Informatics Journal March 2018:66-91.

[9] Paramythis A, Weibelzahl S, Masthoff J. Layered evaluation of interactive adaptive systems: framework and formative methods. User Modeling and User-Adapted Interaction 2010;20:383-453.

[10] Lindgren H, Kampik T, Guerrero E, Blusi M, Nieves JC. Argumentation-based Health Information Systems : A Design Methodology. IEEE Intelligent Systems 2021;36,2:72-80.

[11] Weck S. A Conceptual Model of Behavior Change Progress for the Application within Coaching Systems to Support Sustainable Lifestyle Changes. [Dissertation]. Umeå University; 2021.

[12] Janols R, Lindgren H. A method for co-designing theory-based behaviour change systems for health promotion. Studies in Health Technology and Informatics 2017;235:368-72.

[13] Lindgren H, Guerrero E, Jingar M, Lindvall K, Ng N, Richter Sundberg L, et al. The STAR-C Intelligent Coach: a Cross- Disciplinary Design Process of a Behaviour Change Intervention in Primary Care. Studies in Health Technology and Informatics 2020;273:203-208.

[14] Wright AJ, Norris E, Finnerty AN et al. Ontologies relevant to behaviour change interventions: a method for their development. Wellcome Open Res 2020;5:126

[15] Alfaifi Y. An Ontology to Support Behaviour Change Interventions: Barriers to Activity. Doctoral dissertation, University of Liverpool; 2020. 